Granda Vera, J.; Barbero Álvarez, J.C. y Cortijo Cantos, A. (2019). Determinación de preíndices en pádel mediante análisis cinemático / Determining Pre-cues in Paddle Tennis by Using a Kinematic Analysis. Revista Internacional de Medicina y Ciencias de la Actividad Física y el Deporte vol. 19 (73) pp. 1-18 Http://cdeporte.rediris.es/revista/revista73/artdeterminacion991.htm DOI: http://doi.org/10.15366/rimcafd2019.73.001

\title{
ORIGINAL
}

\section{DETERMINACIÓN DE PREÍNDICES EN PADEL MEDIANTE ANÁLISIS CINEMÁTICO}

\section{DETERMINING PRE-CUES IN PADDLE TENNIS BY USING A KINEMATIC ANALYSIS}

\author{
Granda Vera, J. ${ }^{1}$; Barbero Alvarez, J.C. ${ }^{2}$ y Cortijo Cantos, A. ${ }^{3}$ \\ 1 Titular de Universidad. Universidad de Granada (España) jgranda@ugr.es \\ 2 Profesor Contratado Doctor Universidad de Granada (España) jcba@ugr.es \\ ${ }^{3}$ Profesora de Centro de Menores (Consejería de Bienestar Social-Ciudad Autónoma de Melilla) \\ (España) adisl_cc@hotmail.com
}

Clasificación de la Unesco: 2406.04 Biomecánica / Biomechanics; 6106.09

Procesos de Percepción / Perception Processes

Clasificación Consejo de Europa: 3. Biomecánica del Deporte/Sport

Biomechanics of Sport;12. Aprendizaje Motor/Motor Learning

Recibido 8 de marzo de 2017 Received March 8, 2017

Aceptado 16 de noviembre de 2017 Accepted November 16, 2017

\section{RESUMEN}

El objetivo es determinar la existencia de indicadores visuales (preíndices) en el golpe de derecha desde el fondo de pista tras rebote en pared en padel para cada una de las posibles direcciones (derecha, centro e izquierda) mediante análisis cinemático. Han participado en el estudio cinco jugadores expertos de padel. Los valores encontrados para altura de la mano y la posición del talón izquierdo en el momento de inicio y final del movimiento se revelan como las zonas corporales que más información podrían proporcionar como pre-índices informacionales para el jugador en defensa $(r=, 896, p<, 001$ y $r=, 777, p<, 001)$. Los datos confirman la existencia de tres patrones de movimiento en virtud de la dirección elegida (máxima altura de la mano y mayor ángulo del talón izquierdo respecto al plano transversal del cuerpo cuando la bola se dirige a la parte izquierda de la cancha, tomado como referencia el jugador que golpea).

PALABRAS CLAVES: Preíndices, padel, análisis biomecánico. 


\begin{abstract}
Using a kinematic analysis, this study aims to find out the existence of visual signals (pre-cues) in drive strokes made from the back of the court after the ball hits a wall, considering each possible direction (right, centre and left). Five expert paddle tennis players took part in the study. The body areas that could be most useful to the defensive player as informative pre-cues $(r=.896 p<.001 \mathrm{y}$ $r=.777 p<.001$ ) were those related to the hand height and the position of the left heel, at the beginning and at the end of the movement. These data confirm the existence of three movement patterns according to the chosen direction (maximum hand height and most open left heel angle in relation to the transverse plane of the body when the ball goes to the left half of the court, with the striking player as reference).
\end{abstract}

KEYWORDS: pre-cues, paddle tennis, biomechanical analysis.

\title{
1. INTRODUCCIÓN
}

La habilidad de predecir las acciones de los oponentes es crucial para el desempeño en muchos deportes, particularmente cuando la incertidumbre y las restricciones espaciotemporales son relevantes (Williams, Ford, Eccles, \& Ward, 2011).

El padel es un deporte de raqueta enmarcado dentro de los denominados de carácter abierto, donde de forma continuada y en un breve espacio de tiempo el jugador debe tomar diferentes decisiones, ante las posibles alternativas que se presentan para cada una de las situaciones del juego, estando todo ello mediatizado por la presión temporal delimitada por la trayectoria y velocidad de la pelota, por lo que dichas decisiones y alternativas han de tomarse de forma muy rápida.

Dicho deporte puede describirse como una mezcla de tenis y squash, con un sistema de puntuación similar al tenis y adaptaciones de las reglas, tales como que el servicio deberá efectuarse golpeando la bola como máximo a la altura de la cadera o que la pelota podrá ser golpeada después de contacto en la pared de fondo o paredes laterales antes de dar el segundo bote en el suelo.

La pista de juego mide $20 \mathrm{~m}$. de largo por $10 \mathrm{~m}$. de ancho, dividida por una red de $0,88 \mathrm{~m}$. de altura en el centro de la pista dando lugar a dos zonas iguales de $10 \mathrm{~m}$. x $10 \mathrm{~m}$. Las paredes laterales y de fondo (de material de metacrilato en las pistas reglamentarias) tienen una altura de $3 \mathrm{~m}$. (ver figura 1). 


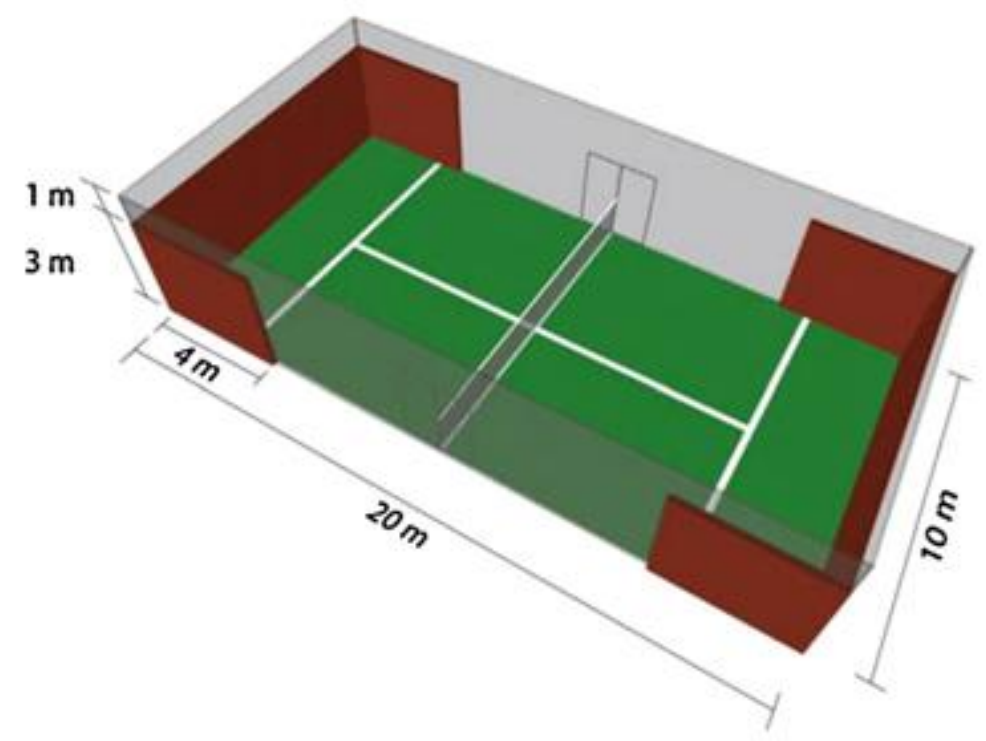

Figura 1. Dimensiones de la pista de padel

El conjunto de golpes que se pueden efectuar se agrupan en tres categorías: golpes directos, golpes indirectos (después de contacto con la pared) y servicio. En un estudio llevado a cabo por González (2006) un 31,8\% de los golpes efectuados correspondían a golpes indirectos, de los cuales un 20,16\% fueron golpes de derecha (forehand).

Carrasco, Romero, Sañudo, y de Hoyo, (2011) encontraron que el golpe más frecuentemente utilizado durante el juego fue la volea $(25,57 \%$ de los golpes totales realizados). A una frecuencia menor, apreciaron el revés (15,57\%), la bandeja $(12,45 \%)$ y el golpe de derecha $(11,80 \%)$. Finalmente, el lob fue el golpe menos utilizado durante la competición de paddle (2,95\%). En cuanto a los golpes indirectos, el golpe de derecha fue el golpe más utilizado $(20,16 \%)$, mientras que el revés mostró una frecuencia de $8,36 \%$, siendo el lob de nuevo el golpe indirecto menos utilizado, representando sólo una frecuencia de 1,80\% del total de los golpes indirectos totales realizados.

De este conjunto de golpes indirectos, el más utilizado fue el golpeo de derecha de la bola tras rebote en la pared de fondo. Este dato es importante, porque conocer la frecuencia de cada uno de los golpes utilizados, ayuda a comprender su importancia técnica y táctica en el desarrollo del juego y su importancia en el resultado final (Blompvist, Luhttanen \& Laakso, 1998).

Para confirmar estos datos, y justificar la realización del presente estudio, se llevó a cabo un análisis cuantitativo de los golpes efectuados en la final de un torneo internacional de padel (III Torneo Internacional Ciudad de Melilla, 2007), en el que participaban los jugadores situados en las cuatro primeras posiciones del ranking mundial, recogiendo los datos mediante un registro observacional, encontrando que, en relación a los golpes indirectos, el golpe más frecuente fue el golpeo de la bola tras rebote en la pared de fondo con un total de 52 golpes a 
lo largo del partido (36\% de golpeos a la derecha del jugador que golpea, un $49 \%$ al centro de la pista y un $15 \%$ a la izquierda del jugador que golpea).

\subsection{LA ANTICIPACIÓN PERCEPTIVA EN EL DEPORTE}

Numerosos estudios señalan que una de las habilidades que claramente inciden en el mejor desempeño de los expertos sobre los noveles es la capacidad de anticipar las acciones del oponente.

Las personas son capaces de predecir futuros eventos en base a la observación de las acciones de otras personas, una habilidad que ha sido estudiada de forma amplísima en el ámbito del deporte. En deportes de raqueta, los jugadores expertos son capaces de predecir exitosamente las intenciones del oponente antes de que ocurra el evento clave tales como el contacto entre la raqueta y la bola (Williams, Ward, Knowles, \& Smeeton, 2002).

Se piensa que la anticipación es resultado de la interacción dinámica entre numerosas habilidades perceptivo-cognitivas (Williams, et al, 2011). Estas habilidades perceptivo-cognitivas incluyen la capacidad de identificar estructuras y familiaridades en secuencias de juego (North, Ward, Ericsson, \& Williams, 2011) y extraer señales de avance durante la preparación del movimiento de su oponente (Abernethy, Zawi, \& Jackson, 2008).

Se han usado diferentes modelos para indagar sobre la capacidad de anticipar la acción del oponente, señalando estos estudios que una de las cuestiones en las que se concreta esta capacidad para anticiparse es atender y discriminar la información que presentan los indicios de avance posturales (Williams y Grant, 1999). El uso de indicios de avance se refiere a la habilidad de un deportista para hacer predicciones exactas basadas en la información contextual disponible desde la etapa inicial de una secuencia de acción (Abernethy, 1987). Este modelo difiere del que sugiere que los deportistas expertos son capaces de usar las expectativas o probabilidades situacionales para facilitar la anticipación en el deporte, referida a la posibilidad de usar su base de conocimiento superior para eliminar algunos eventos porque son altamente improbables y pueden establecer una jerarquía de probabilidades en los eventos que permanecen, pudiendo reducir la incertidumbre relativa a que eventos ocurrirán (incertidumbre del evento) y cuando acontecerá (Granda \& Alemany, 2002:160).

En concreto, en los deportes de raqueta como el padel, la investigación se ha basado mayoritariamente en el uso de las señales/indicios de avance, centrándose gran parte de su atención en la forma en que los jugadores utilizan estos indicios de avance observando al oponente con el fin de disponer del tiempo suficiente para desplazarse en la pista para llegar al punto de interceptación requerida para poder devolver la bola. Esta predicción acerca de la dirección de la bola tras el golpe del adversario se basa, en la mayoría de las situaciones, en la información derivada de hechos que ocurren antes de que el 
oponente realmente golpee la pelota (Abernethy, Gill, Parks, \& Packer, 2001; Williams, Ward, Knowles, \& Smeeton, 2002). Es preciso señalar, que en el caso del padel, la distancia entre oponentes es menor (dimensiones de la pista más pequeña) que en el caso del tenis, lo que concede una mayor importancia a la capacidad de anticipación perceptiva en el jugador en defensa y su influencia en la toma de decisiones consecuente.

Dada la velocidad de la bola que caracteriza este conjunto de deportes, la tarea fundamental del jugador en defensa será predecir, tan pronto como sea posible, la dirección y altura de la bola enviada por el adversario, ya que al igual que en otros deportes caracterizados como abiertos (gran nivel de incertidumbre en la decisión - respuesta a adoptar) (Granda, Mingorance, Barbero, Hinojo, Mohamed y Reyes, 2008 determinar los indicadores o preíndices que permitan al jugador conocer de forma anticipada la acción a realizar por el oponente y aumentar, por tanto, el tiempo para decidir la respuesta motora, se convierte en un factor de gran influencia en el desempeño deportivo.

A este respecto, la anticipación solo es posible si se puede constatar la existencia de diferencias entre características que se mantienen invariantes durante la realización de un determinado golpe (como el que se analiza en el presente estudio). En este sentido Troje (2002) y Westhoff, and Troje (2007) sugieren que las características dinámicas del movimiento son más relevantes que las de tipo anatómico a la hora de predecir la dirección y altura de la bola del oponente.

La investigación relativa a deportes de raqueta muestra que las limitaciones de tiempo son tales que la recogida de información del patrón de movimiento del oponente es crucial en el rendimiento experto (Glencross y Cibich 1977). Al menos dos fuentes principales de información para facilitar la acción anticipatoria pueden estar a disposición del ejecutante: (i) la información derivada específicamente del patrón de movimiento pre-contacto de la raqueta con la bola por parte del oponente, y (ii) la información más genérica relacionada con las probabilidades situacionales dada la situación específica en que se lleva a cabo el golpe por el adversario.

La evidencia disponible hasta la fecha en los deportes de raqueta sugiere que puede haber una relación estrecha entre la experiencia y la capacidad de recoger información acerca de la cinemática de la acción que se está produciendo por parte del adversario. En primer lugar, los expertos utilizan las fuentes de información más proximales durante la ejecución (brazo, así como información de la posición de la raqueta), lo cual es consistente con la noción de que su ventaja se debe a una capacidad superior para evaluar la evolución cinemática proximal - distal de la acción que están observando. Asimismo, estudios comparando las estrategias visuales de expertos y novatos en tenis encontraron que los expertos fijaron durante más tiempo su visión en las zonas de la cabeza, tronco y cadera mientras que los noveles lo hicieron en la posición de la raqueta (Ward, Williams \& Bennett, 2002). Estos mismos autores resaltan que otra de las grandes diferencias entre expertos y novatos puede centrarse en 
la forma en que ambos usan la información cinemática.

Estos hallazgos confirman que la estructura temporal en los golpeos de la bola en tenis (situación idéntica a la que se realiza en padel) informa a los oponentes sobre la futura posición espacial de la bola pero no clarifica cómo esta información está plasmada en los patrones de movimiento (Huys, Smeeton, Hodges, Beek, \& Williams, 2008).

\subsection{APLICACIÓN DEL ANÁLISIS BIOMECÁNICO AL ESTUDIO DE LA ANTICIPACIÓN PERCEPTIVA}

El avance de la tecnología en los últimos años ha permitido llevar a cabo detallados análisis cinemáticos tridimensionales de las acciones de golpeo de una bola con la raqueta. Estos métodos biomecánicos han permitido investigar los mecanismos subyacentes utilizados en la ejecución de las habilidades que implican una acción de golpeo con una raqueta (Lees, 2003).

Las técnicas de análisis tridimensionales de las habilidades de raqueta han permitido determinar datos relativos a los valores angulares de las articulaciones participantes, velocidades lineales y angulares y velocidades de bola en el servicio de tenis (Elliott, Marsh, \& Blanksby, 1986; Papadopoulis, Emmanouilidou, \& Prassas, 2000), el golpe de revés en tenis (Elliott et al., 1989a), el golpe de derecha en tenis (Elliott et al., 1989b) y la volea en el tenis (Elliott, Overheu, \& and Marsh, 1988).

La cinemática de todos los golpes en los deporte de raqueta sigue una secuencia predecible próximo-distal en el reclutamiento de los segmentos participantes en la acción, comenzando con un paso hacia delante de la pierna contralateral, seguido de la acción de la cadera y la rotación del tronco y, a continuación seguida, en orden, por los movimientos segmentarios de la parte superior del brazo, antebrazo y mano (Elliott et al, 1986; Woo y Chapman 1991).

Ward et al., (2002) en un estudio con un jugador de tenis experto encontraron diferencias entre los patrones de movimientos de los diferentes golpes de tenis, incluyendo en estas diferencias variaciones en parámetros como el valor máximo en los ángulos de las articulaciones consideradas, aunque con diferencias mínimas en los parámetros analizados en función de la dirección de la bola.

Los patrones de movimiento encontrados mediante el análisis cinemático puede ser descompuesto en términos de unas pocas estructuras 0 componentes. Troje (2002) encontró que los patrones de marcha de hombres y mujeres pueden ser descompuestos en cuatro componentes rítmicos mediante el uso del análisis de componentes principales en un análisis factorial.

Huys, et al., (2008) usaron una metodología similar a la de Troje para estudiar la ejecución de golpes de tenis con diferentes direcciones. Hallaron 
estructuras dinámicas subyacentes en la ejecución de los golpes en tenis y que todas las regiones corporales contribuían a la generación de estas estructuras dinámicas, con pequeñas variaciones en los grados de libertad de un golpe a otro.

A partir de estos hallazgos Huys, Cañal-Bruland, Hagemann, Beek, and Smeeton (2009) examinaron la importancia de la información localizada en determinadas estructuras dinámicas (raqueta, hombros, caderas y piernas) cuando los jugadores tratan de anticipar la dirección de los golpes en tenis, mediante la utilización del paradigma de oclusión visual. Los resultados mostraron que los jugadores anticipan la dirección del golpe a través de una aproximación perceptiva global más que en estructuras más localizadas, especialmente los jugadores más habilidosos o expertos.

Alonso y Argudo (2011) llevaron a cabo un análisis notacional sobre el rendimiento del saque en frontenis olímpico. Entre las conclusiones destacar el hecho de que el saque se mostraba más efectivo cuando se realizaba con un desplazamiento a la derecha y que los saques que provocaban más dificultades para su devolución eran los rodados que buscaban la pared lateral 3, aunque no eran los más utilizados

Basándose en las investigaciones precedentes en este mismo ámbito, el objetivo de este estudio es determinar la existencia de patrones cinemáticos en la secuencia de movimientos del golpe de derecha desde el fondo de pista tras rebote en pared en padel para cada una de las posibles direcciones (derecha, centro e izquierda) mediante análisis cinemático tridimensional que pudieran ser utilizados como señales/preíndices de avance para su posterior aplicación al entrenamiento y mejora de la capacidad de anticipación perceptiva en la respuesta defensiva en situaciones reales de juego.

\section{MATERIAL Y MÉTODO}

\subsection{PARTICIPANTES}

Han participado en el estudio cinco jugadores de padel de élite y expertos (ver tabla 1).

Tabla 1. Características de cada uno de los participantes en el estudio

\begin{tabular}{|c|c|c|c|c|c|}
\hline & Categoría/Ranking & Sexo & Edad & Altura & Peso \\
\hline Participante 1 & Internacional/Campeón del mundo & $\mathrm{V}$ & 32 & $176 \mathrm{~cm}$ & $75 \mathrm{Kg}$ \\
\hline Participante 2 & $\begin{array}{l}\text { Internacional/Subcampeón } \\
\text { mundo }\end{array}$ & V & 30 & $177 \mathrm{~cm}$ & $74 \mathrm{~kg}$ \\
\hline Participante 3 & Nacional/8 ranking nacional & $\mathrm{H}$ & 24 & $178 \mathrm{~cm}$ & $73 \mathrm{~kg}$ \\
\hline Participante 4 & Nacional/7 ranking nacional & $\mathrm{V}$ & 25 & $172 \mathrm{~cm}$ & $68 \mathrm{~kg}$ \\
\hline Participante 5 & Nacional/12 ranking nacional & $\mathrm{V}$ & 23 & $175 \mathrm{~cm}$ & $72 \mathrm{~kg}$ \\
\hline
\end{tabular}


Todos los participantes fueron previamente informados del propósito y objetivos del estudio y dieron su consentimiento para el registro y análisis de los datos incluidos en el presente trabajo.

\subsection{MATERIAL}

Se han utilizados tres cámaras Casio EXF1 para el registro de las acciones de golpeo realizadas por los participantes en el estudio y el software Kinescan (IBV) versión 8.3 para la digitalización y análisis cinemático del movimiento.

\subsection{PROCEDIMIENTO}

Una vez informados los jugadores y dado su consentimiento para su participación en el estudio, se procedió a la grabación de los golpeos en unas instalaciones municipales, siendo la pista utilizada de medidas reglamentarias. Previamente a la filmación de los golpeos se grabó un sistema de referencia o estructura de geometría y dimensiones conocidas, que soporta unos marcadores ubicados de forma fija y de los que se conoce su posición con gran precisión. En nuestro estudio, utilizamos un sistema con forma de cubo, cuyas dimensiones eran $2 \times 2 \times 2$ metros.

Se utilizaron tres cámaras de vídeo que filmaron a una frecuencia de 250 f.p.s. (fotogramas por segundo). Dos de ellas se ubicaron de forma que sus ejes ópticos estuvieran entre $90^{\circ}$ y $100^{\circ}$ y de manera que existieran el menor número de marcadores ocultos para facilitar la posterior digitalización y una en la otra mitad de la pista donde se realizaban los golpes, de manera frontal respecto a la situación de los participantes para registrar la dirección de la bola.

Las imágenes se capturaron a formato digital, convirtiéndose en ficheros AVI con los que se llevó a cabo el proceso de digitalización.

Una vez filmado el sistema de referencia, el jugador participante se situaba en el centro geométrico de su zona (mitad de la pista) y un jugador colaborador lanzaba una bola desde el campo contrario (acción de globo o lob) que rebotaba en la pared posterior una vez hubiera golpeado en el suelo, realizando el jugador participante un desplazamiento hacia atrás para acercarse a dicha pared y realizar el golpe de derecha desde el fondo de pista tras rebote en pared. El jugador de forma aleatoria y voluntaria elegía la dirección de la bola (derecha, centro o izquierda de la pista) tras ser golpeada. Una cámara auxiliar grababa la acción de golpeo desde una perspectiva frontal para discriminar dicha dirección y relacionar posteriormente valores cinemáticos encontrados (patrones de movimiento) y dirección de la bola.

Cada participante realizó un total de 50 repeticiones de la acción analizada. Del total de acciones grabadas (350) solo se han considerado para el estudio un total de 72 , aquellas valoradas como acciones de un nivel experto de ejecución, determinada esta condición por tres entrenadores expertos de padel. 
La técnica de adquisición de datos fue la fotogrametría en 3D mediante vídeo. Esta técnica consiste en la obtención de las coordenadas de un punto, a partir de la filmación simultánea del evento con dos o más cámaras y posterior tratamiento de las imágenes.

Una vez filmadas las acciones, se procedió a la digitalización de los datos, proceso que se llevó a cabo en cuatro fases: calibración, digitalización de la escena, suavizado de las coordenadas, obtención de los resultados y gestión de gráficas.

El modelo mecánico utilizado fue el propuesto por Clauser (1969) y Zatsiorsky, Seluyanov, and Chugunova (1990). Se digitalizaron de forma manual 25 puntos (sistema + implemento) para cada fotograma, es decir un sistema más implemento formado por el jugador y la pala, más la bola. Los parámetros inerciales corporales fueron tomados de De Leva (1996) y finalmente los datos resultantes fueron filtrados con funciones splines de quinto orden (Woltring, 1985).

Las variables de estudio han sido de dos tipos: espaciales (altura de la mano de golpeo, la distancia de separación entre ambos pies medida en los talones y la posición de los talones derecho e izquierdo) y angulares (ángulos de la cadera derecha y rodillas derecha e izquierda), tanto en el momento de inicio del movimiento como en el momento del golpeo de la bola. La codificación la hizo un solo observador y para asegurar la fiabilidad del registro, se eligieron aleatoriamente 5 golpes del conjunto de golpes válidos, que fueron registrados por el observador en dos ocasiones (con una semana de diferencia y sin conocer los valores obtenidos en el primer registro en el momento de hacer el segundo). Se alcanzó un valor de fiabilidad intraobservador del $98 \%$.

Una vez obtenidos los valores cinemáticos concurrentes para cada una de las tres direcciones y las relaciones entre los diferentes segmentos corporales, se visionaron dos partidos de padel correspondientes a las finales de dos torneos internacionales, en las que participaban los cuatro mejores jugadores del mundo en ese momento, para establecer la correspondencia entre lo encontrado en el análisis cinemático y las acciones en la realidad del juego.

\subsection{ANALISIS DE LOS DATOS}

Para el análisis de los datos se utilizó el paquete estadístico SPSS V.20, sometiendo los valores encontrados a la prueba de correlación de Pearson para determinar la existencia de relaciones entre ellos, para posteriormente realizar una análisis de regresión, seleccionando el modelo de regresión lineal, por considerar que era el que mejor se adecuaba a la estructura de los datos, una vez comparados diferentes modelos de este estadístico. 


\section{RESULTADOS}

En la tabla 2 se presentan los valores descriptivos promedio de las variables de estudio en el momento del comienzo de la acción (máxima acción de rotación de la mano de golpeo contra la dirección de golpeo) y en el momento de finalizar la acción (contacto de la pala con la bola) encontrados en el conjunto de golpes válidos analizados.

Tabla 2. Estadísticos descriptivos de las variables estudiadas

\begin{tabular}{lrr}
\hline & Media & \multicolumn{1}{c}{$S D$} \\
\hline Distancia entre talones comienzo (distalc) & 0,59 & 0,11 \\
Distancia entre talones final (distalf) & 0,63 & 0,11 \\
Altura mano golpeo comienzo (altmanderc) & 2,42 & 0,33 \\
Altura mano golpeo final (altmanderf) & 2,32 & 0,37 \\
Talón izquierdo comienzo (talizqc) & 1,96 & 0,44 \\
Talón izquierdo final (talizqf) & 1,97 & 0,43 \\
Talón derecho comienzo (talderc) & 1,78 & 0,41 \\
Talón derecho final (talderf) & 1,76 & 0,41 \\
Ángulo rodilla izquierda comienzo (angrodizqc) & 141,24 & 46,94 \\
Ángulo rodilla izquierda final (angrodizqf) & 145,70 & 37,89 \\
Ángulo rodilla derecha comienzo (angcadderc) & 156,25 & 6,88 \\
Ángulo rodilla derecha final (angcadderf) & 161,97 & 10,59 \\
Ángulo cadera derecha comienzo (angrodderc) & 140,56 & 7,53 \\
Ángulo cadera derecha final angrodderf & 144,51 & 10,86 \\
\hline
\end{tabular}

En la tabla 3 se presentan los valores encontrados al someter los datos al estadístico R de Pearson.

Tabla 3. Valores relativos a las correlaciones significativas entre las variables de estudio

\begin{tabular}{|c|c|c|c|c|c|c|c|c|}
\hline & distalf & talizqc & talizqf & talderf & $\begin{array}{l}\text { angrodi } \\
\text { zqf }\end{array}$ & $\begin{array}{l}\text { angcad } \\
\text { derf }\end{array}$ & $\begin{array}{l}\text { angrod } \\
\text { derc }\end{array}$ & $\begin{array}{l}\text { angrod } \\
\text { derf }\end{array}$ \\
\hline \multirow[t]{2}{*}{ distalc } & 0,698 & & & & & & & \\
\hline & 0,000 & & & & & & & \\
\hline \multirow[t]{2}{*}{ altmanderc } & & 0,896 & 0,891 & & & & & \\
\hline & & 0,000 & 0,000 & & & & & \\
\hline \multirow[t]{2}{*}{ altmanderf } & & 0,778 & 0,777 & & & & & \\
\hline & & 0,000 & 0,000 & & & & & \\
\hline \multirow[t]{2}{*}{ talizqc } & & & 0,988 & & & & & \\
\hline & & & 0,000 & & & & & \\
\hline \multirow[t]{2}{*}{ talderc } & & & & 0,997 & & & & \\
\hline & & & & 0,000 & & & & \\
\hline \multirow[t]{2}{*}{ angrodizqc } & & & & & 0,953 & & & \\
\hline & & & & & 0,000 & & & \\
\hline \multirow[t]{2}{*}{ angcadderc } & & & & & & 0,652 & 0,418 & 0,418 \\
\hline & & & & & & 0,000 & 0,022 & 0,022 \\
\hline \multirow[t]{2}{*}{ angrodderc } & & & & & & & & 0,584 \\
\hline & & & & & & & & 0,001 \\
\hline
\end{tabular}


Como se comprueba en la tabla 3 , existen relaciones significativas entre las variables relativas a posiciones espaciales y entre las variables angulares entre sí, no encontrando ninguna relación significativa entre las variables espaciales y angulares.

Dado que la discriminación de valores angulares como preíndices es algo ciertamente complejo en una situación real de juego, y al no encontrar relación entre éstos y los valores de las variables espaciales, se sometieron solamente los valores de las variables espaciales al modelo de análisis de regresión lineal, presentando los resultados encontrados en la tabla 4.

Tabla 4. Valores en la regresión lineal

\begin{tabular}{|c|c|c|c|c|c|}
\hline Variable predictora & Variable dependiente & $\mathrm{R}$ & R cuadrado & $F$ & Sig. \\
\hline $\begin{array}{l}\text { Alt mano de golpeo } \\
\text { comienzo }\end{array}$ & $\begin{array}{l}\text { Talón } \\
\text { comienzo }\end{array}$ & 0,896 & 0,803 & 113,83 & 0,000 \\
\hline $\begin{array}{l}\text { Alt mano de golpeo } \\
\text { comienzo }\end{array}$ & Talón izquierdo final & 0,891 & 0,793 & 107,51 & 0,000 \\
\hline Alt mano de golpeo final & $\begin{array}{l}\text { Talón } \\
\text { comienzo }\end{array}$ & 0,778 & 0,605 & 42,82 & 0,000 \\
\hline Alt mano de golpeo final & Talón izquierdo final & 0,777 & 0,604 & 42,66 & 0,000 \\
\hline
\end{tabular}

\section{DISCUSIÓN}

Este estudio tenía como objetivo tratar de determinar la existencia de un patrón motor (cinemático) específico para cada una de las posibles direcciones de la bola en una situación de golpeo en padel, mediante el uso de técnicas tridimensiones, con objeto de poder incluir en los entrenamientos estos hallazgos para facilitar la acción anticipatoria de los jugadores en situación defensiva ante esta acción.

Del conjunto de datos analizados, los valores encontrados para altura de la mano y la posición del talón izquierdo en el momento de inicio y final del movimiento se revelan como las zonas corporales que más información podrían proporcionar como pre-índices informacionales para el jugador en defensa, relación que se mantiene altamente significativa entre los valores de ambas variables al comienzo y al final de la acción (ver figuras 2, 3 y 4). 


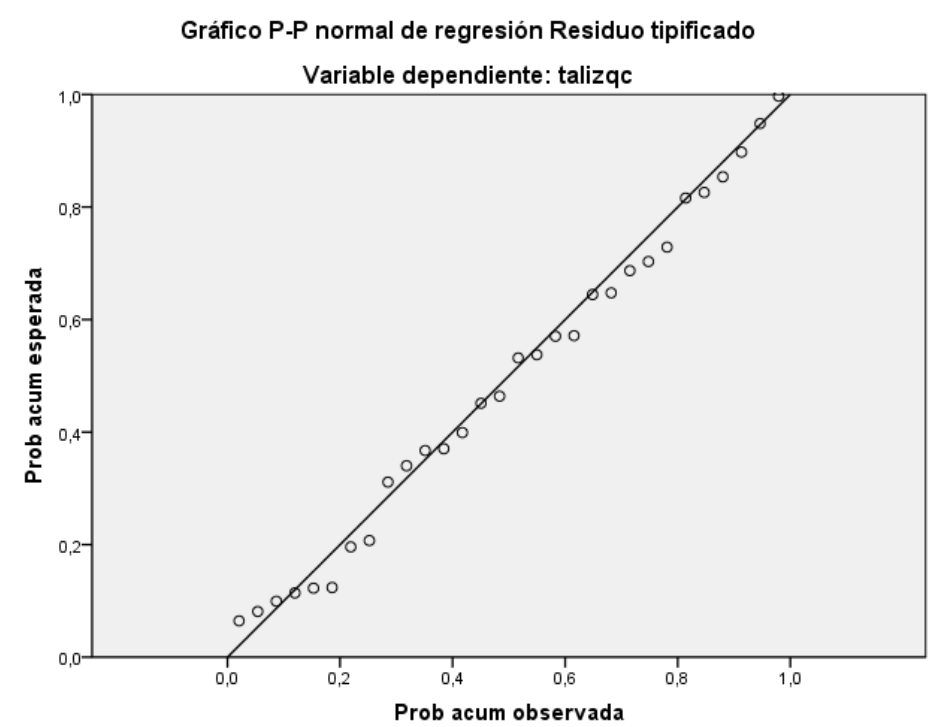

Figura 2. Gráfica de los valores encontrados en la regresión lineal de las variables altura de la mano de golpeo comienzo (altmanderc) y posición del talón izquierdo comienzo (talizqc)

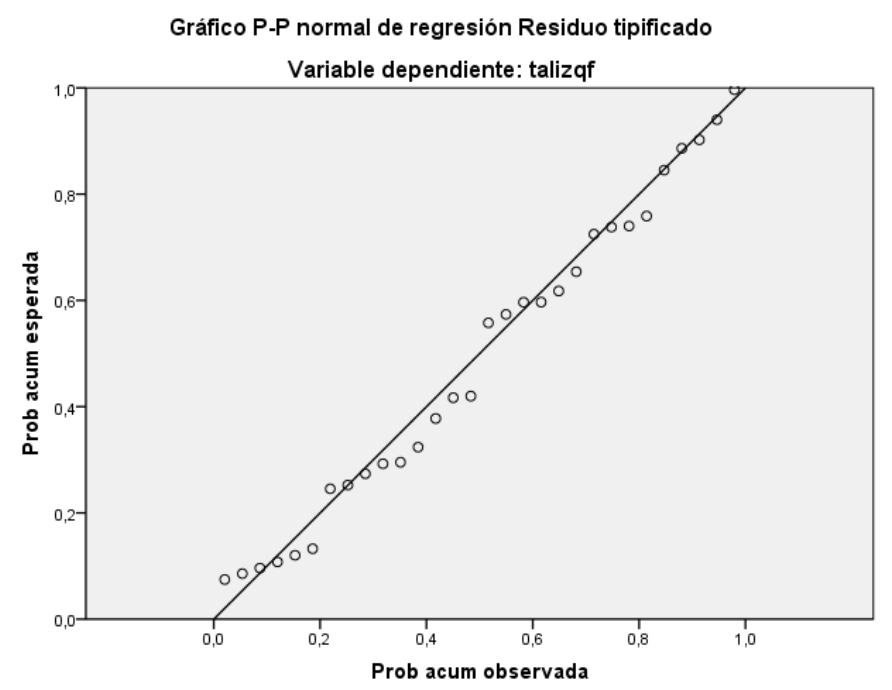

Figura 3. Gráfica de los valores encontrados en la regresión lineal de las variables altura de la mano de golpeo comienzo (altmanderc) y posición del talón izquierdo final (talizqf) 


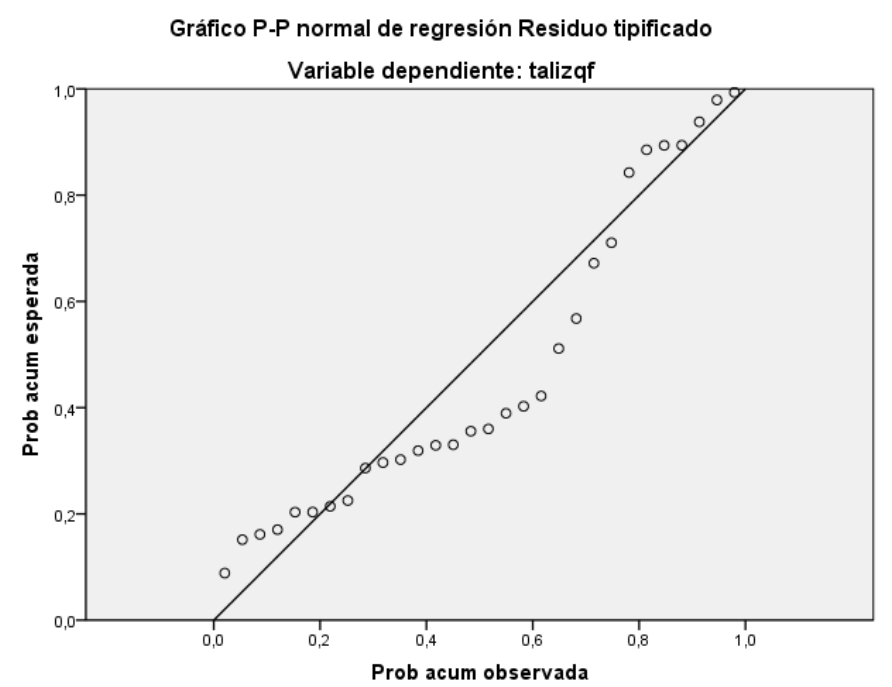

Figura 4. Gráfica de los valores encontrados en la regresión lineal de las variables altura de la mano de golpeo final (altmanderf) y posición del talón izquierdo final (talizqf)

Los datos parecen confirmar la existencia de tres patrones de movimiento para cada una de estas dos variables en virtud de la decisión del jugador que golpea respecto a la dirección a enviar la bola, adoptando diferentes puntos de partida en las variables estudiadas según la dirección elegida. Así se puede comprobar como a una mayor altura de la mano en el momento del golpeo de la bola corresponde una posición del pie izquierdo (talón) más abierta (lejana) (dirección de la bola a la izquierda del jugador que golpea) respecto al eje longitudinal de la pista, mientras que a alturas más bajas corresponden valores posicionales del talón izquierdo más cerrado respecto a dicho eje longitudinal (ver figura 5) (dirección de la bola a la derecha del jugador que golpea). En el caso de golpes al centro de la pista se comprueba que el talón del pie izquierdo se halla situado encima del eje longitudinal del jugador. Estos resultados son coincidentes con los encontrados por Ward et al., (2002) en un estudio con jugadores expertos de tenis, hallando diferencias en los patrones de movimiento de los diferentes golpes de tenis estudiados.

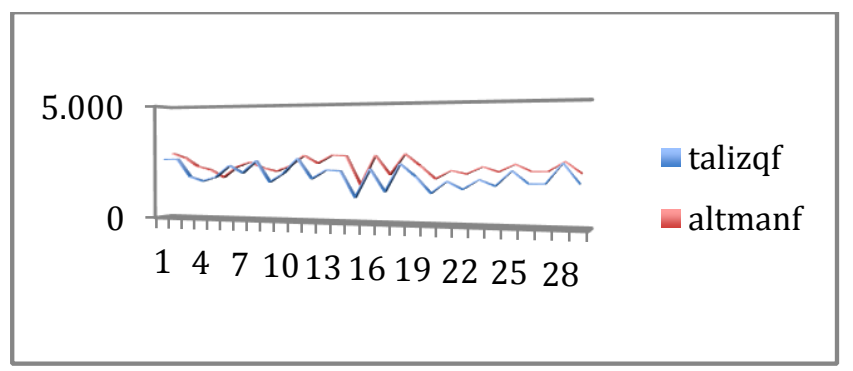

Figura 5. Valores de la altura de la mano de golpeo y del talón izquierdo en el momento del golpeo de la bola

Tal como sugiere la evidencia disponible hasta la fecha en los deportes de raqueta, los expertos utilizan las fuentes de información más proximales durante la ejecución (brazo, así como información de la posición de la raqueta), lo cual es consistente con la noción de que su ventaja se debe a una capacidad 
superior para evaluar la evolución cinemática proximal - distal de la acción que están observando (Elliott et al, 1986; Woo y Chapman 1991). Esta relación próximo-distal (raqueta - pie izquierdo) se constata en esta acción de golpeo, lo que sugiere que puede ser información relevante en la mejora de la capacidad anticipatoria del jugador defensor en este tipo de situaciones.

Este hallazgo permitiría introducir situaciones de aprendizaje que mejoren el uso de las habilidades perceptivo-cognitivas de jugadores de padel en proceso de formación, las cuales incluyen la capacidad de identificar estructuras y familiaridades en secuencias de juego (North, et al., 2011) y les permitan extraer señales de avance durante la preparación del movimiento de su oponente (Abernethy, et al., 2008).

Los resultados encontrados en el presente estudio son también coincidentes con lo señalado por Huys, et al., (2009) quienes resaltaron la importancia de la información localizada en determinadas estructuras dinámicas (raqueta, hombros, caderas y piernas) cuando los jugadores tratan de anticipar la dirección de los golpes en tenis, mediante la utilización del paradigma de oclusión visual.

Para establecer el valor ecológico de estos hallazgos, se analizaron dos partidos de padel de alta competición (finales de torneos internacionales Torneos Internacional de Melilla 2007 y 2008), en los que participaban las dos mejores parejas del mundo en ese momento. Un total de 106 situaciones de golpeo de la bola tras impacto en la pared fueron encontradas, con un total de 40 situaciones $(37,22 \%)$ donde la bola fue dirigida hacia la derecha de la pista (desde la perspectiva del jugador que golpea), un total de 50 situaciones $(47,1 \%)$ donde la bola fue dirigida al centro de la pista y un total de 12 situaciones (16\%) donde la bola fue dirigida hacia la zona izquierda del campo desde la perspectiva del jugador que golpea la bola.

Del conjunto de situaciones registradas, en un $89 \%$ de las veces se encontró que los jugadores mostraban el patrón cinemático reseñado anteriormente en función de la dirección que la bola tomaba una vez golpeada (ver figuras 6, 7 y 8). En el resto de situaciones (11\%) los jugadores rompían esta relación patrón cinemático-dirección de la bola mediante acciones propias de sujetos de gran maestría que les permiten llevar a cabo acciones contrarias al modelo cinemáticamente correcto. 


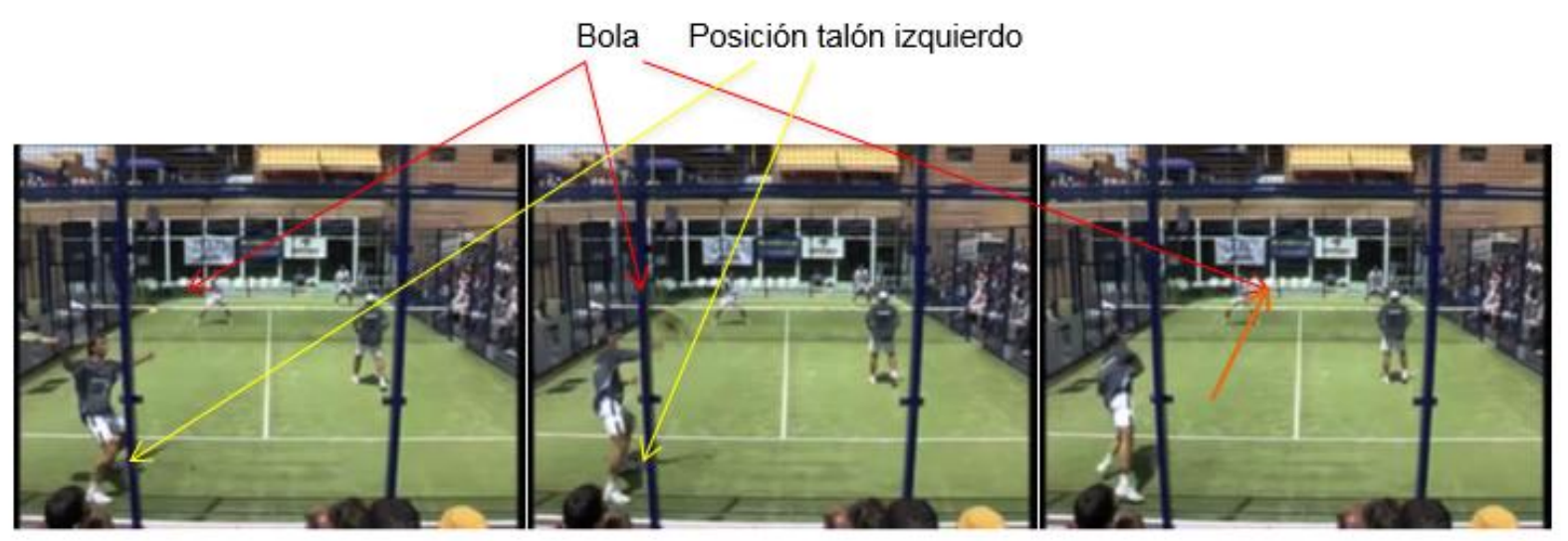

Figura 6. Secuencia golpeo dirección bola lado izquierdo de la pista (perspectiva del jugador que golpea la bola)

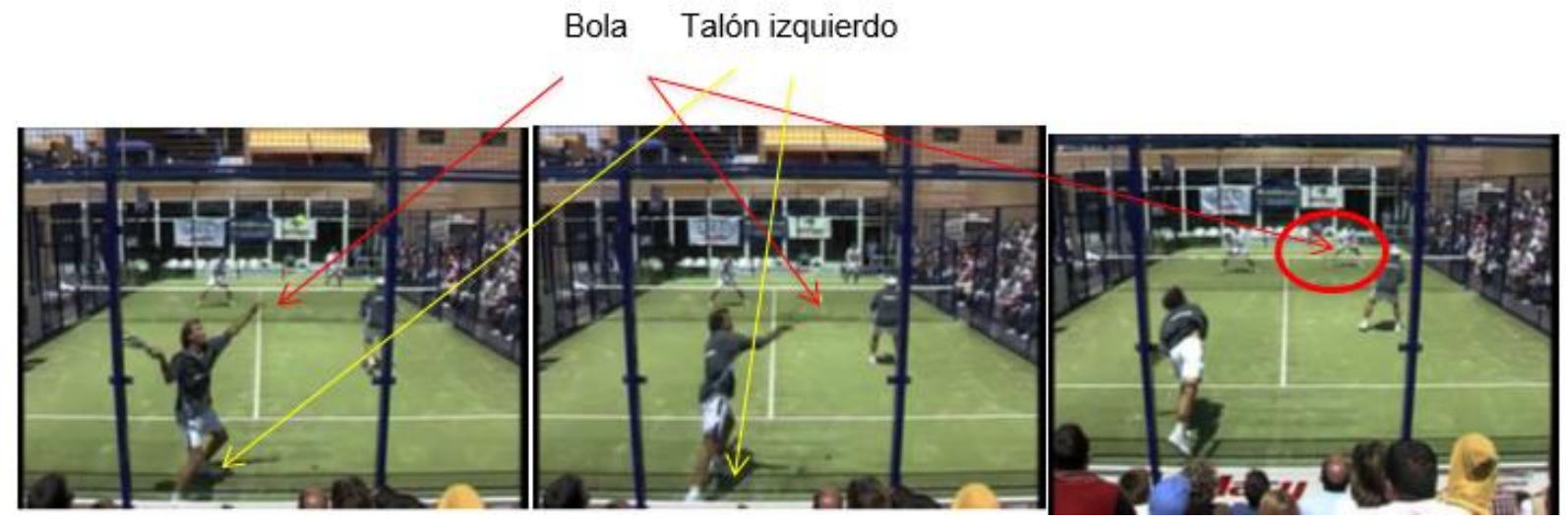

Figura 7. Secuencia golpeo dirección bola lado derecho de la pista (perspectiva del jugador que golpea la bola)

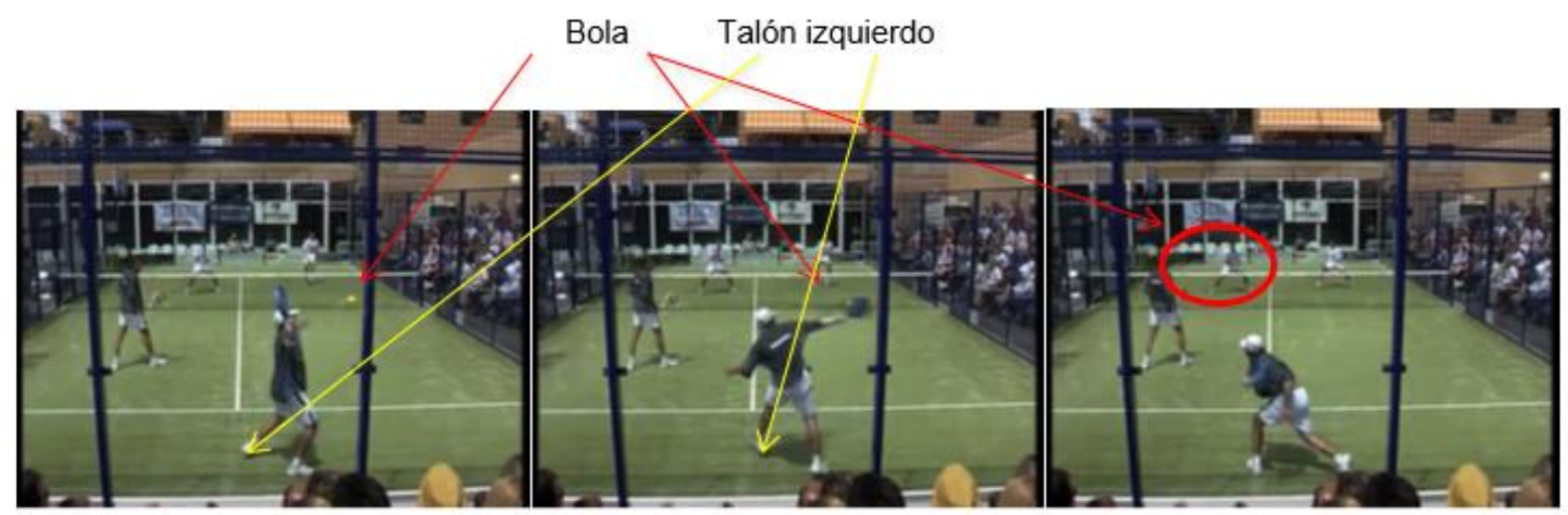

Figura 8. Secuencia golpeo dirección bola centro de la pista (perspectiva del jugador que golpea la bola)

\section{CONCLUSIONES}

En conclusión, la aplicación del análisis cinemático para determinar la existencia de informaciones posturales (señales de avance) en situaciones 
deportivas abiertas, que permitan su posterior inclusión en los procesos de aprendizaje de jugadores noveles, se muestra como una herramienta de gran potencial en la identificación de las claves que faciliten la respuesta anticipatoria y un más rápido y eficaz desempeño en situaciones reales de juego. Los resultados encontrados parecen confirmar la existencia de diferencias dinámicas en los patrones de golpeo en virtud de la dirección de la bola, posibilitando su uso en la mejora de la capacidad de anticipación perceptiva de jugadores de padel, ya que ésta solo es posible si se puede constatar la existencia de diferencias entre características que se mantienen invariantes durante la realización de un determinado golpe (Troje, 2002 y Westhoff and Troje, 2007).

A partir de estos resultados, será necesario proseguir en estos estudios desde la perspectiva del jugador defensor, para determinar si los preíndices posturales encontrados en esta investigación son usados por los jugadores en situaciones reales de juego para tratar de anticipar la dirección de la bola y cobrar ventaja en la acción de respuesta.

\section{REFERENCIAS BIBLIOGRÁFICAS}

Abernethy, B. (1987). Anticipation in sport: A review. Physical Education Review, 10, 5-16. https://doi.org/10.1068/p2872

Abernethy, B., Gill, D. P., Parks, S. L., \& Packer, S. T. (2001). Expertise and the perception of kinematic and situational probability information. Perception,30, 233-252. https://doi.org/10.1068/p5340

Abernethy, B., Zawi, K., \& Jackson, R. C. (2008). Expertise and attunement to kinematic constraints. Perception,37, 931-948.

Alonso Roque, J.I. y Argudo Iturriaga, F. (2011). Análisis notacional informatizado del rendimiento del saque en frontenis olímpico. Revista Internacional de Medicina y Ciencias de la Actividad Física y el Deporte, vol.11 (42), pp. 421-439.

Blompvist, M., Luhttanen P., \& Laakso, L. (1998). Validation of a notational analysis system in badminton. Journal Human Movements Studies,35, 137-150.

Carrasco, L., Romero, S., Sañudo, B. \& de Hoyo, M. (2011). Game analysis and energy requirements of paddle tennis competition. Analyse du jeu et exigences physiologiques dans la pratique du padel en competition. Science \& Sports,26, 338-344. https://doi.org/10.1016/j.scispo.2010.12.016

Clauser, C.E., McConville, J.T. , Young, J.W. (1969). Weight, volume, and center of mass of segments of the human body. AMRL-TR-69-70. Aerospace Medical Research Laboratory, Wright-Patterson Air Force Base, Ohio. https://doi.org/10.21236/AD0710622

De Leva, P. 1996). Adjustments to Zatsiorsky-Seluyyanov's segment inertia parameters. Journal Biomechanics, $19 \quad$ (9), 1223-1230. https://doi.org/10.1016/0021-9290(95)00178-6

Elliott, B. C., Marsh, A., \& Blanksby, B. (1986). A three-dimensional cinematographic analysis of the tennis serve. International Journal of Sport 
Biomechanics,2, 260-270. https://doi.org/10.1123/ijsb.2.4.260

Elliott, B. C., Overheu, P. R. and Marsh, A. P. (1988). The service line and net volleys in tennis: a cinematographic analysis. Australian Journal of Science and Medicine in Sport,20 (1), 10-18.

Elliott, B. C., Marsh, A. P., \& Overheu, P.R. (1989a). The topspin backhand drive in tennis: a biomechanical analysis. Journal of Human Movement Studies, 16, 1-16.

Elliott, B. C., Marsh, T., \& Overheu, P. (1989b). A biomechanical comparison of the multisegment and single unit topspin forehand drives in tennis. International Journal of Sports Biomechanics,5, 350-364. https://doi.org/10.1123/ijsb.5.3.350

Glencross, D. J., \& Cibich, B. J. (1977). A decision analysis of games skills. Australian Journal of Sports Medicine, 9, 72-75.

González-Carvajal C. (2006). Escuela de pádel. Del aprendizaje a la competición amateur. Madrid: Tutor.

Granda, J., y Alemany, I. (2002). Manual de Aprendizaje y Desarrollo Motor. Barcelona: Paidós Educación Física.

Granda, J., Mingorance, A., Barbero, J.C., Hinojo, D., Mohamed, N., y Reyes, $M^{a}$.T. (2008). Effects of and additional computer simulation training programme and/or on-court specific instructions on advance cue detection in basketballers. International Journal of Sport Psychology, 39 (3), 257277

Huys, R., Smeeton, N. J., Hodges, N. J., Beek, P. J., \& Williams, A. M. (2008). On the dinamyc information underlying visual anticipation skill. Perception \& Psychophysics, 7(7), 1217-1234. https://doi.org/10.3758/PP.70.7.1217

Huys, R., Cañal-Bruland, R., Hagemann, N., Beek, P. J., and Smeeton, N. J. (2009). Global information pickup underpins anticipation of tennis shot direction. Journal of Motor Behavior,41(2), 158-170. https://doi.org/10.3200/JMBR.41.2.158-171

James, N., Bradley, C., \& Mellalieu, S. (2006). The disguise and deception of an international squash player. Journal of Sports Sciences, 24(4), 340-341.

Lees, A. (2003). Science and the major racket sports: a review. Journal of Sports Sciences,21, 707-732. https://doi.org/10.1080/0264041031000140275

North, J. S., Ward, P., Ericsson, A., \& Williams, A. M. (2011). Mechanism underlying skilled anticipation and recognition in a dynamic and temporally constrained domain. Memory, 19, 155-168. https://doi.org/10.1080/09658211.2010.541466

Papadopoulis, C., Emmanouilidou, M. \& Prassas, S. (2000). Kinematic analysis of the service stroke in tennis. In S. Haake \& A. O. Coe (Eds.). Tennis Science and Technology (pp. 383-388). Oxford: Blackwell.

Troje, N. F. (2002). Decomposing biological motion: A framework for analysis and synthesis of human gait patterns. Journal of Vision,2, 371-387. https://doi.org/10.1167/2.5.2

Ward, P., Williams, A. M., \& Bennett, S. J. (2002). Visual search and biological motion perception in tennis. Research Quarterly for Exercise and Sport, 73, 107-112. https://doi.org/10.1080/02701367.2002.10608997

Westhoff, C., \& Troje, N. F. (2007). Kinematic cues for person identification from 
biological motion. Perception \& Psychophysics,69, 241-253. https://doi.org/10.3758/BF03193746

Williams, A. M., \& Grant, A. (1999). Training perceptual skill in sport. International Journal of Sport Psychology,30, $194-220$.

Williams, A. M., Ward, P., Knowles, J. M., \& Smeeton, N. J. (2002). Anticipation skill in a real-world task: Measurement, training, and transfer in tennis. Journal of Experimental Psychology: Applied, 8, 259-270. https://doi.org/10.1037/1076-898X.8.4.259

Williams, A.M., Ford, P.R., Eccles, D. W., \& Ward, P. (2011). Perceptual-cognitive expertise in sport and its acquisition: Implication for applied cognitive psychology. Applied Cognitive Psychology, 25, 432-442. https://doi.org/10.1002/acp.1710

Woo, H., \& Chapman, A. (1991). A 3D kinematic analysis of the squash forehand stroke', in R. Marshall, G. Wood, B. Elliott, T. Ackland, \& P. McNair (Eds). Proceedings of the XIIlth International Congress on Biomechanics (pp. 147-148). Perth, WA: University of Western Australia.

Zatsiorsky, V. M., Seluyanov, V. N., \& Chugunova, L. G. (1990). Methods of determining mass-inertial characteristics of human body segments. In G.G. Chernyi \& S.A. Regirer. Contemporary Problems of Biomechanics (pp. 272-291). USA: CRC Press.

Número de citas totales / Total references: 31

Número de citas propias de la revista / Journal's own references: 1

Rev.int.med.cienc.act.fís.deporte - vol. 19 - número 73 - ISSN: 1577-0354 